\title{
Clinical and molecular characterization of Italian patients affected by Cohen syndrome
}

\author{
Eleni Katzaki - Chiara Pescucci - Vera Uliana - Filomena Tiziana Papa · Francesca Ariani · \\ Ilaria Meloni - Manuela Priolo · Angelo Selicorni - Donatella Milani · Rita Fischetto · \\ Maria Elena Celle · Rita Grasso · Bruno Dallapiccola · Francesco Brancati · Marta Bordignon · \\ Romano Tenconi $\cdot$ Antonio Federico $\cdot$ Francesca Mari $\cdot$ Alessandra Renieri $\cdot$ Ilaria Longo
}

Received: 15 May 2007/Accepted: 30 September 2007 / Published online: 8 November 2007

(C) The Japan Society of Human Genetics and Springer 2007

\begin{abstract}
Cohen syndrome is an autosomal recessive disorder with variability in the clinical manifestations, characterized by developmental delay, visual disability, facial dysmorphisms and intermittent neutropenia. We described a cohort of 10 patients affected by Cohen syndrome from nine Italian families ranging from 5 to 52 years at assessment. Characteristic age related facial changes were well documented. Visual anomalies, namely retinopathy and myopia, were present in $9 / 10$ patients (retinopathy in $9 / 10$ and myopia in $8 / 10$ ). Truncal obesity has been described in all patients older than 6 years $(8 / 8)$. DNA samples from all patients were analyzed for mutations in $\mathrm{COH} 1$ by DHPLC. We detected $15 \mathrm{COH}$
\end{abstract}

Electronic supplementary material The online version of this article (doi:10.1007/s10038-007-0208-4) contains supplementary material, which is available to authorized users.

E. Katzaki - C. Pescucci - V. Uliana - F. T. Papa - F. Ariani ·

I. Meloni · F. Mari · A. Renieri $(\bowtie) \cdot$ I. Longo

Medical Genetics, Department of Molecular Biology,

University of Siena, V. Le Bracci 2, 53100 Siena, Italy

e-mail: renieri@unisi.it

M. Priolo

Medical Genetics Hospital of Reggio Calabria,

Reggio Calabria, Italy

A. Selicorni · D. Milani

Pediatric Unit, University of Milan, Milan, Italy

R. Fischetto

U.O. Metabolic Disease-Medical Genetics,

P.O.Giovanni XXIII-A.O.U. Policlinico Consorziale,

Bari, Italy

M. E. Celle

Child Neuropsychiatric Unit, G. Gaslini Institute,

University of Genova, Genova, Italy alterations most of them were truncating mutations, only one being a missense change. Partial gene deletions have been found in two families. Most mutations were private. Two were already reported in the literature just once. A single base deletion leading to p.T3708fs 3769 , never reported before, was found in three apparently unrelated families deriving from a restricted area of the Veneto's lowland, between Padova town and Tagliamento river, in heterozygous state. Given the geographical conformation of this region, which is neither geographically or culturally isolated, a recent origin of the mutation could be hypothesized.

Keywords Cohen syndrome - $\mathrm{COHI}$ - Heterogeneity · DHPLC · Founder effect

R. Grasso

IRCCS MEDEA, Bosisio Parini, Lecco, Italy

B. Dallapiccola $\cdot$ F. Brancati

IRCCS CSS, Mendel Institute, Rome, Italy

B. Dallapiccola

Department of Experimental Medicine and Pathology,

La Sapienza University, Rome, Italy

M. Bordignon · R. Tenconi

Clinical Genetics and Epidemiology,

University of Padova, Padova, Italy

A. Federico

Department of Neurological and Behavioural Sciences,

University of Siena, Siena, Italy 


\section{Introduction}

Cohen syndrome (OMIM \#216550) is an autosomal recessive condition firstly described in 1973 by Cohen et al. (1973). It is characterized by non-progressive psychomotor retardation usually of mild to severe degree, characteristic facial features, hypotonia and intermittent neutropenia (Carey and Hall 1978; Cohen et al. 1973; Norio et al. 1984). The peculiar craniofacial features of Cohen syndrome include microcephaly, downslanting and wave shaped palpebral fissures, short philtrum and prominent upper central incisors (Carey and Hall 1978; Cohen et al. 1973; Norio et al. 1984).

In 2003, mutations in the $\mathrm{COH} 1$ gene were identified as cause of Cohen syndrome in the Finnish population (Kolehmainen et al. 2003). The $\mathrm{COH}$ gene maps to chromosome 8q22 and consists of 62 exons that encode for a potential transmembrane protein presumably involved in vesicle mediated sorting and intracellular protein transport (Kolehmainen et al. 2003; Velayos-Baeza et al. 2004). Five different splicing isoforms with an apparent differential expression are known. Two transcripts of about 2 and $5 \mathrm{~kb}$ have been identified either in fetal brain, lung, liver, and kidney, and in all adult tissues analyzed. In the adult, a larger transcript of approximately $12-14 \mathrm{~kb}$ shows high levels of expression in prostate, testis, ovary, and colon tissues, while its expression is very low in brain tissue (Kolehmainen et al. 2003). This variety of transcripts may suggest different tissue and/or timing dependent protein functions.

Phenotypic spectrum in the Finnish patients is highly homogeneous and molecular analysis revealed a strong founder effect with the presence of a common ancestral mutation causative of the majority of cases (Hennies et al. 2004). More recently, Cohen syndrome was found to be associated with mutations in the $\mathrm{COH} 1$ gene in different populations with a broader clinical spectrum than the Finnish subtype (Falk et al. 2004; Hennies et al. 2004; Kolehmainen et al. 2003; Mochida et al. 2004; Seifert et al. 2006). To clarify the clinical and molecular features associated with Cohen syndrome in the Italian population, we describe here 10 patients from nine Italian families in which $\mathrm{COH} 1$ mutations have been identified.

\section{Materials and methods}

\section{Patients}

Ten patients (eight sporadic subjects and a familial case represented by two sisters) with a clinical suspect of Cohen syndrome were included in this study after obtaining written informed consent. All patients attended a Clinical
Genetics Department in Italy and were diagnosed by experienced Italian clinical geneticists. All cases were of Italian ancestry with age at assessment ranging from 5 to 52. There were eight non-consanguineous families having all one affected child, with the exception of one family with two affected sisters, and one consanguineous family with one affected child. Blood samples were collected from each proband and from the parents whenever possible after signed informed consent. Clinical features of each patient are summarized in Table 1 and shown in Fig. 1.

\section{Molecular analysis}

Genomic DNA was extracted using QIAamp DNA blood maxi kit, according to the manufactures' protocol (Qiagen, Hilden, Germany). PCR amplification of the 62 exons was performed using primers previously reported by Kolehmainen et al. 2003 except of 2, 8, 19, 22, 26, 29, 38, 47, 49, 56 and 57 (Table 2). Mutation analysis was performed by denaturing high performance liquid chromatography (DHPLC) using the Transgenomic WAVE ${ }^{\mathrm{TM}}$ (Transgenomic, San Jose, CA, USA) and genomic sequencing analysis. PCR products were denatured at $95^{\circ} \mathrm{C}$, reannealed at $65^{\circ} \mathrm{C}$ for $10 \mathrm{~min}$, and cooled at $4{ }^{\circ} \mathrm{C}$ to generate heteroduplexes. The optimal column temperature for fragments analysis was calculated using the WaveMaker Software (Transgenomic, San Jose, CA, USA) (DHPLC conditions are available on request). PCR products resulting in abnormal DHPLC profiles were purified and sequenced on both strands by using PE Big dye terminator cycle sequencing kit on an ABI Prism 310 genetic analyser (PE Applied Biosystems, Foster City, CA, USA).

The sensitivity of the DHPLC technique is high when searching for heterozygous mutations. In order to detect homozygous mutations we performed mutational screening in the proband and in one of the parents of all our cases. In those patients where parents DNA samples were not available, we performed mutational screening mixing control DNA with patients' samples (1:1 ratio).

To evaluate the $\mathrm{COH} 1$ gene dosage, we used seven Custom TaqMan Gene Expression Assays by design, which provide pre-designed primers-probe set for real-time qPCR experiments (Applied Biosystems, https://products. appliedbiosystems.com). The TaqMan probes were designed in exon 3 , exon 16 , exon 17 , exon 20 , exon 24 , exon 44 and exon 55 (qPCR primers and probes are reported on S1). Quantitative PCR was carried out using an ABI prism 7000 (Applied Biosystems, Foster City, California) in a 96-well optical plate with a final reaction volume of $50 \mu \mathrm{l}$. A total of $100 \mathrm{ng}$ of DNA $(10 \mu \mathrm{l})$ was dispensed in each of the four sample wells for quadruplicate reactions. Thermal cycling conditions included a pre- 
run of $2 \mathrm{~min}$ at $50^{\circ} \mathrm{C}$ and $10 \mathrm{~min}$ at $95^{\circ} \mathrm{C}$. Cycle conditions were 40 cycles at $95^{\circ} \mathrm{C}$ for $15 \mathrm{~s}$ and $60^{\circ} \mathrm{C}$ for $1 \mathrm{~min}$, according to the TaqMan Universal PCR Protocol (PE Applied Biosystems, Foster City, CA, USA). The TaqMan Universal PCR Master Mix and Microamp reaction tubes were supplied by Applied Biosystems. The starting copy number of the unknown samples was determined using the comparative $\mathrm{Ct}$ method, as previously described (Ariani et al. 2004).

In order to predict eventual changes to the protein caused by amino acid substitutions, we used the PredictProtein Server (http://cubic.bioc.columbia.edu/ predictprotein/), a multiple service for sequence analysis and structure prediction, which uses a total of other 14 prediction programs.

\section{Results}

The coding region of the $\mathrm{COH} 1$ gene was analyzed by DHPLC in all 62 exons. Fourteen $\mathrm{COH} 1$ heterozygous mutations were identified in the 10 Cohen patients included in the study (Table 1). Despite the presence of consanguinity in one family, homozygous mutations were not found. For those patients where only one mutation was found, we performed sequencing analysis and we identified one more pathogenic change in case 4 .

Ten different point mutations were found (Fig. 2). Most of them were truncating: 4 nonsense, 4 frame-shift, 1 splice site mutation (Table 1). Only one was a missense change and it was not seen in 100 chromosomes from control samples. Most mutations are private. Two were already reported in the literature in one family: p.Q3772X, p.R2535X, (Hennies et al. 2004). A single base deletion in exon 58 (c.11125delC) leading to p.T3708fs3769, never reported before, was found, in heterozygous state, in three apparently unrelated patients (1,2 and 3), coming from a restricted area of the Veneto's lowland between Padova town and Tagliamento river (S2). This mutation leads to a stop codon in position 3,769 of the protein, cutting off the last transmembrane domain, likely giving rise to a non functional COH1 product (http://www.expasy.org accession number Q7Z7G8). In two cases (1 and 2) the mutation was paternally inherited while in the other case (3) it was inherited from the mother.

In order to evaluate the pathogenic role of the missense mutation found in case 6 , we have used a PredictProtein server which is a service for sequence analysis and secondary structure prediction. From the output of this service we have found that the p.A590T substitution seems to
Fig. 1 Features of Italian patients with Cohen syndrome. a Patients 1, 2, 3, 6, 9A and 9B are shown. Signed informed consents were obtained from the parents of the affected children and guardians of affected adults for publication of the images. Note the characteristic facial gestalt of these patients. b Frontal view of patients 4 and 8 . Note the truncal obesity of both cases. c Hands of cases 2, 6, 7 and 8 . The fingers are slender and tapering from the proximal interfalangeal joint a
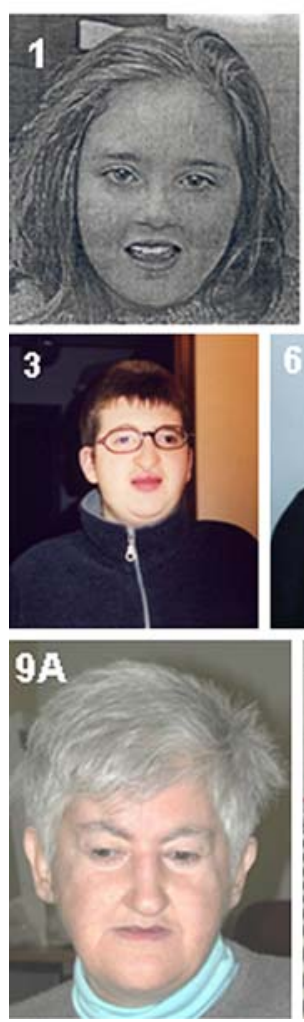
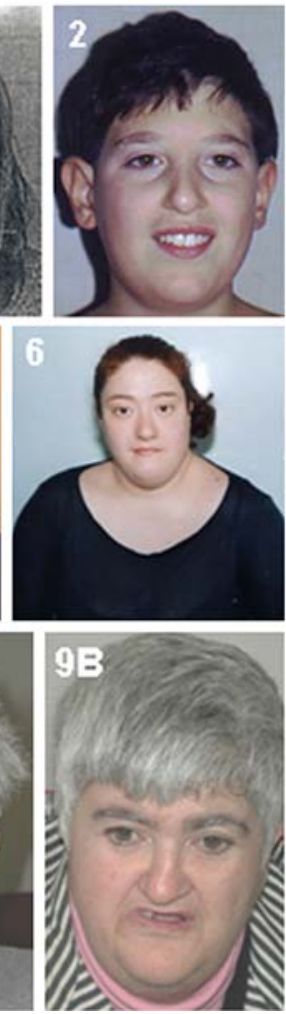

b
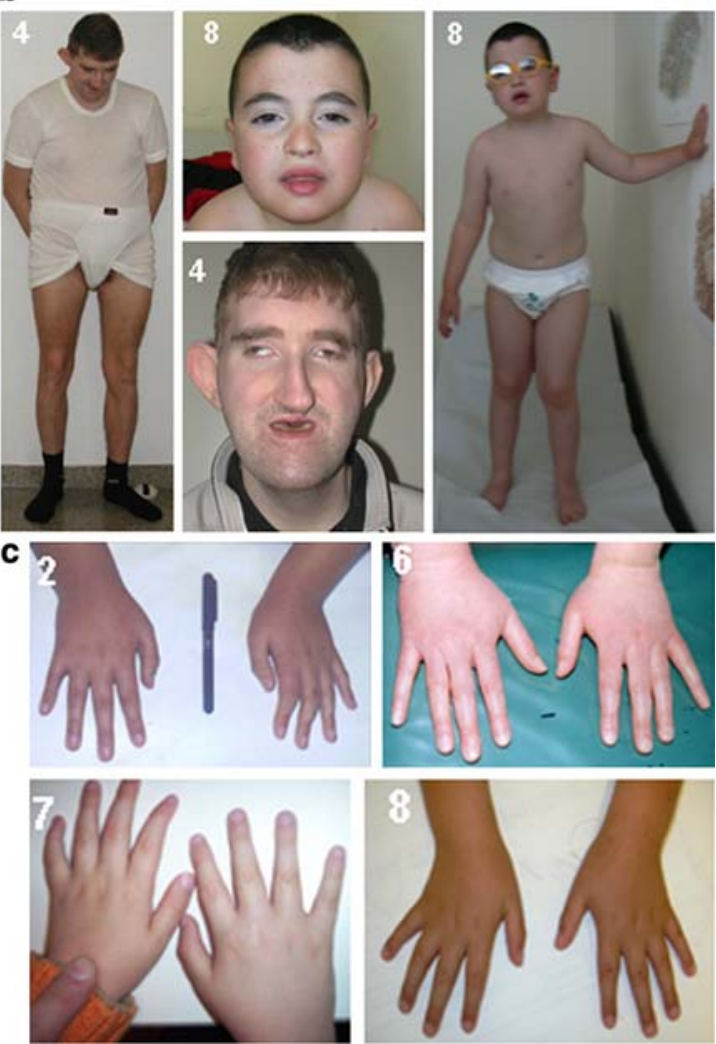


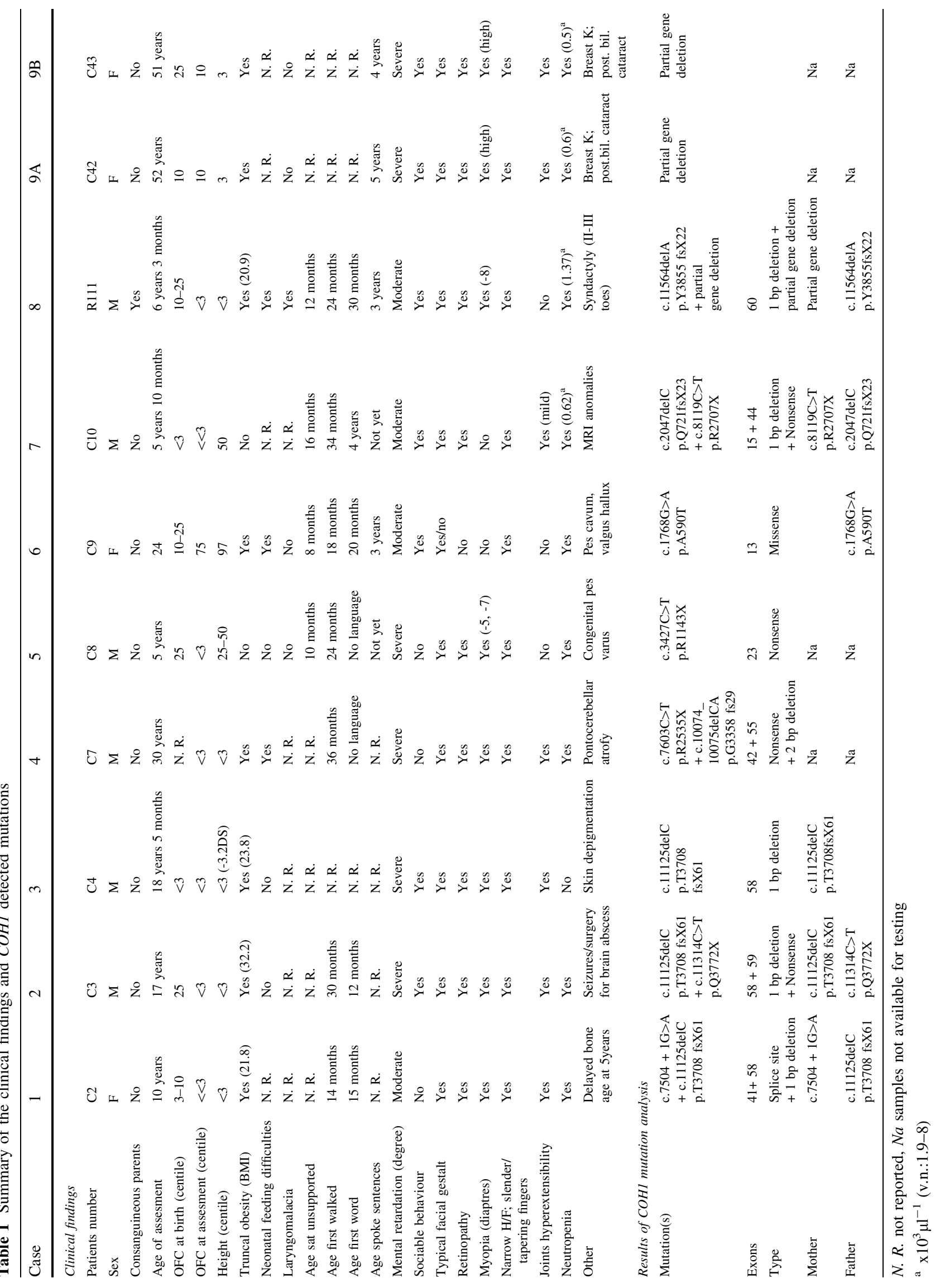




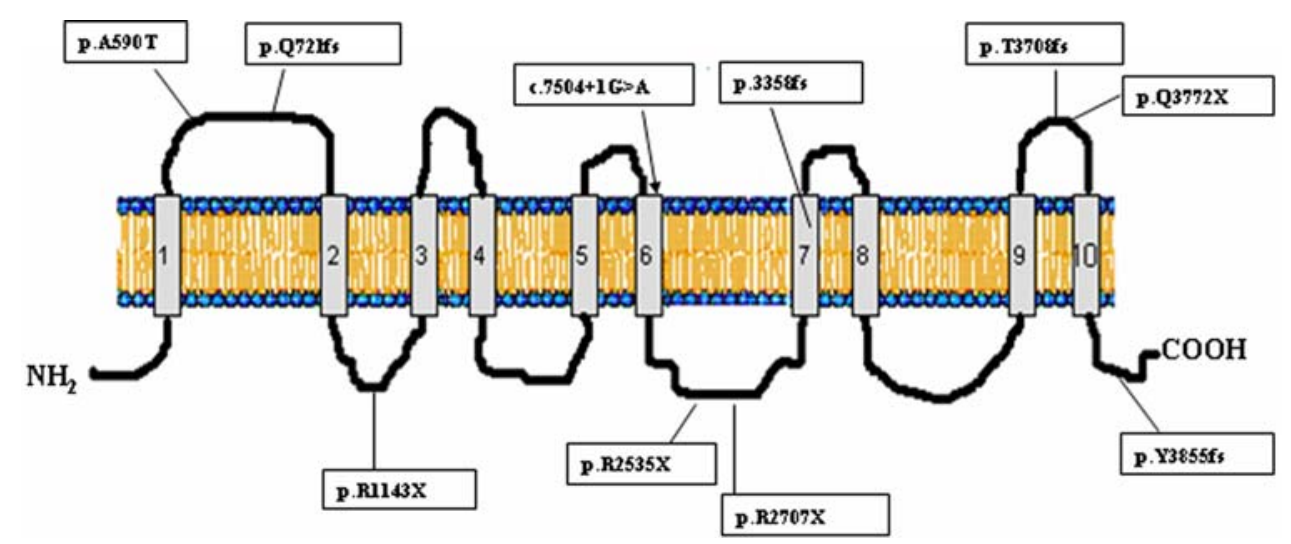

Fig. 2 Schematic description of the predicted $\mathrm{COH} 1$ protein with representation of the transmembrane domains and intracellular and extracellular loops. Mutations are indicated along the protein. An arrow indicates a splice-site mutation named at the DNA level. The others mutations, which are named at the protein level, are indicated with straight lines. The p.Q3772X mutation (cases 1, 2 and 3) creates

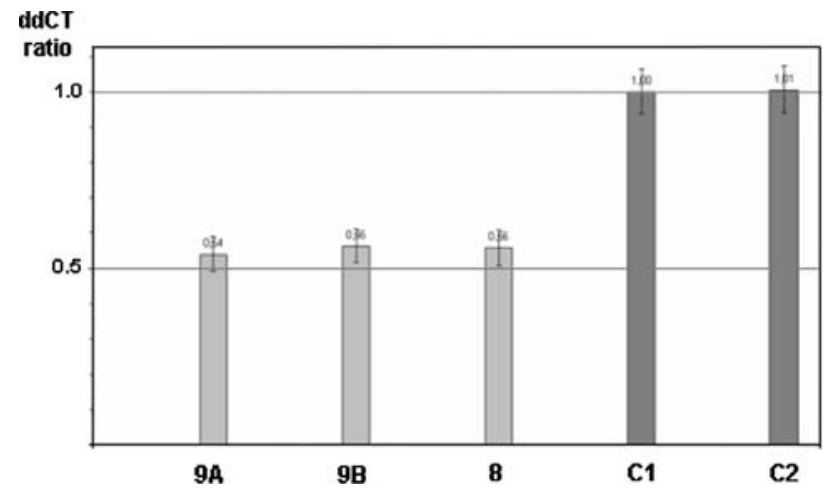

Fig. 3 Real time quantitative PCR results in exon 16. ddCT ratios and standard deviations of two control samples (c1 and c2) and of three patients (9A, 9B and 8). Compared to controls, samples 9A, 9B and 8 show ddCT ratio values of about 0.5 , indicating a $\mathrm{COH} 1$ deletion

influence the protein folding. In particular, the state of the predicted disulfide bonds seems to be altered. For example, the strength of each predicted disulfide bond at cysteines in positions 600 and 648 seems to be decreased. On the other hand, this substitution is predicted to be responsible of the creation of a new disulfide bond at cysteine in position 650 . In addition, this substitution p.A590T seems to alter the helical trasmembrane regions and the difference of positive charges between the internal and the external layer of the membrane. Given all these data we cannot exclude the pathogenic role of this substitution.

No homozygous gene deletions have been identified in our patients. Quantitative PCR using probes on exons 3, 16, 17, 20, 24, 44 and 55 were performed in order to identify whole or partial heterozygous gene deletions. Partial gene deletions were identified in heterozygous state in three a stop codon in position 3772 , cutting off the last transmembrane domain. The frameshift mutation p.Q721fs744X and the nonsense mutation p.R2707X, both leading to premature protein interruption were found in case 7 . The frameshift mutation p.Y3855fs $3877 \mathrm{X}$ was identified in case 8 , in which a partial gene deletion in the other allele has been found

patients belonging to two different families, all three deletions were found using the probe on exon 16 (Fig. 3).

\section{Discussion}

Cohen syndrome is a rare disorder, described for the first time in the Finnish population as an entity with clinical homogeneity. However, in the Italian population there seems to be a more phenotypic variability compared to the Finnish Cohen syndrome patients, confirming what previously reported in other non-Finnish cases.

In our series of 10 patients with Cohen syndrome from 10 families, the age at unsupported sitting was variable from 8 to 16 months (median age 11.5 months) and the first walking alone was variable from 1 to 3 years (median age 25.7 months). The achievement of the abilities to say words or sentences was very variable and one patient (patient 4) has never achieved verbal communication skills (Table 1). With regards to stature, 7 out of 10 patients (70\%) were below the third centile, 2 out of $10(20 \%)$ were within the normal range and one case was at the 97th centile. Microcephaly was present in nearly all patients (7/ 10) and it was always postnatal except in one case. Visual anomalies were found in $9 / 10$ patients (retinopathy in $9 / 10$ and myopia in 8/10). Truncal obesity was present in all patients older than 6 years (8/8). Mental retardation varied from moderate to severe. Typically narrow hands with slender and tapering fingers were present in all patients (see Fig. 1c). Joint hyperextensibility is present in $7 / 10$ patients.

Typical craniofacial features have been observed in all our patients (Fig. 1). Downward slanting eyes with thick eyebrows lending to "china doll" appearance were evident especially in patients 2,3 , and 8 (Fig. 1a, b). Convex 
Table 2 Primers sequences

\begin{tabular}{lll}
\hline Exon number & Forward primer $\left(5^{\prime}>3^{\prime}\right)$ & Reverse primer $\left(5^{\prime}>3^{\prime}\right)$ \\
\hline 2 & - & GATTACCGTCTAAACAAGCTG \\
8 & - & TCTACTGAATGCAAAGCAA \\
19 & ATCAAATAAAGTTGAAATGTTATATTATG & GCAAAACAAGGGAATAATGATAG \\
22 & CGTTTGGTATGTTCTGTG & - \\
26 & CATTTGCATGTAAGATGTGA & CAACAAGAGCAAAACTCTGT \\
$29 \_2$ & ATCTCCTGGTCAGCCCATGA & ATATACCAAACCACAAAGCAC \\
38 & GAACATAATTACAGTCCTAC & TGAAGAACTTCCCCTAAG \\
47 & - & CCCCAGTGCAAGGTTACTTT \\
49 & - & GATCATAAACGCAACTTTAC \\
$56 \_1$ & - & TTCCACGTATAACCGAGCA \\
$56 \_2$ & CATATCCAACAAAGAGTTGG & - \\
57 & - & AAGGAGTGAAGGCATTATTA \\
\hline
\end{tabular}

profile of nose, short philtrum with open mouth and prominent lips were present in patients $1,2,3,4,8$. The facial phenotype of patient 6 is less characteristic, showing some similarities with an atypical patient described by Seifert et al. (Falk et al. 2004; Hennies et al. 2004; Kolehmainen et al. 2003; Mochida et al. 2004; Seifert et al. 2006). Patient 7 was 6 years old at assessment and presented wave-shaped eyelids, a hypotonic facial expression with an open mouth (picture not reported). The mouth had downturned corners and the lower lip was thick and pouting. The philtrum was not so obviously short.

It is worthwhile to note the phenotypic changes from young to adult patients. In childhood the face was round with a full lower lip, the philtrum is short but not excessively, the eyes are often slightly downward slanting with wave-shaped eyelids and the nasal bridge is much less prominent than the adults. With increasing age, the facial gestalt changes. The philtrum becomes very short, the columella further grows giving the impression of a shorter philtrum, the nasal bridge becomes higher, and central incisors may appear prominent; sometimes early loss of teeth may be present contributing to maxillar hypoplasia (see patients 4, 9A and 9B of Fig. 1).

We have tried to classify our cohort of patients from a clinical point of view. We used Kolehmainen criteria since criteria described by Chandler are mainly designed to get a precocious diagnosis in early infancy (Kolehmainen et al. 2004; Chandler et al. 2003). Since the mean age of our patients was 21 years ranging between 5 and 52 years Kolehmainen criteria appear to be more appropriate. Nine out of 10 can be classified as true Cohen patients and 1/10 can be classified as a Cohen like case. The phenotype of this last patient (case 6) is similar to that previously described as Jewish type of Cohen syndrome: stature at 97th centile, head circumference at 75 th centile, absence of ocular involvement, although she has neutropenia which is usually absent in the Jewish type (Kondo et al. 1990; Sack and Friedman 1986).

In this paper we report 10 mutated Cohen patients from nine families; five are compound heterozygotes. A heterozygous mutation has been found in the other five cases. We have missed five mutated alleles. The lack of a second pathogenic mutation can be attributed to the presence of partial deletions not covering exons screened by qPCR or point mutations in yet uncovered alternative exons or in the promoter region. In the patient with the Jewish type of Cohen syndrome we identified only a $\mathrm{COHI}$ heterozygous missense mutation, which could justify the milder phenotype.

Unexpectedly, the proband of the consanguineous family was a genetic compound. In this case (case 8) a frameshift mutation in one allele (c.11564delA) and a partial gene deletion in the other were identified. This is the first report in which gene deletions are investigated at genomic level. In previous papers only a few intragenic deletions were reported and they were identified at cDNA level (Kolehmainen et al. 2004; Seifert et al. 2006).

One mutated allele was a missense mutation. PredictProtein Server predicts that the missense mutations, p.A590T (case 6) probably cause a change in the secondary structure of the $\mathrm{COH} 1$ protein. This mutation is located in the first extracellular loop of the protein and it has never been reported before. In addition, it was not found in 100 chromosomes from control subjects.

In our paper we also found a mutation in three apparently unrelated families coming from a restricted area of the Veneto's lowland (between Padova and Tagliamento). This could lead us to think of a possible founder effect. Given the geographical conformation of this region, which is neither geographically or culturally isolated, a recent origin of the mutation could be hypothesized.

In summary, this is the first report of Cohen syndrome in the Italian population. Our cohort further confirms that 
Cohen syndrome has a distinctive clinical phenotype identifiable not only in the Finnish patients but also in other genetically different patient groups like in ours.

Acknowledgments We thank all the families for their participation in this study.

\section{References}

Ariani F, Mari F, Pescucci C, Longo I, Bruttini M, Meloni I, Hayek G, Rocchi R, Zappella M, Renieri A (2004) Real-time quantitative PCR as a routine method for screening large rearrangements in Rett syndrome: report of one case of MECP2 deletion and one case of MECP2 duplication. Hum Mutat 24(2):172-177

Carey JC, Hall BD (1978) Confirmation of the Cohen syndrome. J Pediatr 93(2):239-244

Chandler KE, Kidd A, Al-Gazali L, Kolehmainen J, Lehesjoki AE, Black GC, Clayton-Smith J (2003) Diagnostic criteria, clinical characteristics, and natural history of Cohen syndrome. J Med Genet 40(4):233-241

Cohen MM Jr, Hall BD, Smith DW, Graham CB, Lampert KJ (1973) A new syndrome with hypotonia, obesity, mental deficiency, and facial, oral, ocular, and limb anomalies. J Pediatr 83(2):280-284

Falk MJ, Feiler HS, Neilson DE, Maxwell K, Lee JV, Segall SK, Robin NH, Wilhelmsen KC, Traskelin AL, Kolehmainen J, Lehesjoki AE, Wiznitzer M, Warman ML (2004) Cohen syndrome in the Ohio Amish. Am J Med Genet A 128(1):23-28

Hennies HC, Rauch A, Seifert W, Schumi C, Moser E, Al-Taji E, Tariverdian G, Chrzanowska KH, Krajewska-Walasek M, Rajab A, Giugliani R, Neumann TE, Eckl KM, Karbasiyan M, Reis A, Horn D (2004) Allelic heterogeneity in the COH1 gene explains clinical variability in Cohen syndrome. Am J Hum Genet 75(1):138-145
Kolehmainen J, Black GC, Saarinen A, Chandler K, Clayton-Smith J, Traskelin AL, Perveen R, Kivitie-Kallio S, Norio R, Warburg M, Fryns JP, de la Chapelle A, Lehesjoki AE (2003) Cohen syndrome is caused by mutations in a novel gene, $\mathrm{COH} 1$, encoding a transmembrane protein with a presumed role in vesicle-mediated sorting and intracellular protein transport. Am J Hum Genet 72(6):1359-1369

Kolehmainen J, Wilkinson R, Lehesjoki AE, Chandler K, KivitieKallio S, Clayton-Smith J, Traskelin AL, Waris L, Saarinen A, Khan J, Gross-Tsur V, Traboulsi EI, Warburg M, Fryns JP, Norio R, Black GC, Manson FD (2004) Delineation of Cohen syndrome following a large-scale genotype-phenotype screen. Am J Hum Genet 75(1):122-127

Kondo I, Nagataki S, Miyagi N (1990) The Cohen syndrome: does mottled retina separate a Finnish and a Jewish type? Am J Med Genet 37(1):109-113

Mochida GH, Rajab A, Eyaid W, Lu A, Al-Nouri D, Kosaki K, Noruzinia M, Sarda P, Ishihara J, Bodell A, Apse K, Walsh CA (2004) Broader geographical spectrum of Cohen syndrome due to COH1 mutations. J Med Genet 41(6):e87

Norio R, Raitta C, Lindahl E (1984) Further delineation of the Cohen syndrome; report on chorioretinal dystrophy, leukopenia and consanguinity. Clin Genet 25(1):1-14

Sack J, Friedman E (1986) The Cohen syndrome in Israel. Isr J Med Sci 22(11):766-770

Seifert W, Holder-Espinasse M, Spranger S, Hoeltzenbein M, Rossier E, Dollfus H, Lacombe D, Verloes A, Chrzanowska KH, Maegawa GH, Chitayat D, Kotzot D, Huhle D, Meinecke P, Albrecht B, Mathijssen I, Leheup B, Raile K, Hennies HC, Horn D (2006) Mutational spectrum of $\mathrm{COH} 1$ and clinical heterogeneity in Cohen syndrome. J Med Genet 43(5):e22

Velayos-Baeza A, Vettori A, Copley RR, Dobson-Stone C, Monaco AP (2004) Analysis of the human VPS13 gene family. Genomics 84(3):536-549 